Article

\title{
Static and Dynamic Responses of Micro-Structured Beams
}

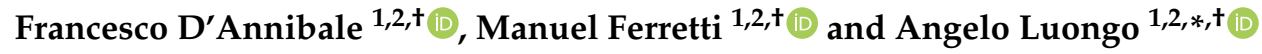 \\ 1 Department of Civil, Construction-Architectural and Environmental Engineering, University of L'Aquila, \\ 67100 L'Aquila , Italy; francesco.dannibale@univaq.it (F.D.); manuel.ferretti@univaq.it (M.F.) \\ 2 International Research Centre for Mathematics and Mechanics of Complex Systems, University of L'Aquila, \\ 67100 L'Aquila, Italy \\ * Correspondence: angelo.luongo@univaq.it; Tel.: +39-0862434521 \\ + These authors contributed equally to this work.
}

Received: 13 August 2020; Accepted: 26 September 2020; Published: 29 September 2020

check for updates

\begin{abstract}
In this study, we developed a one-dimensional Timoshenko beam model, embedded in a 3D space for static and dynamic analyses of beam-like structures. These are grid cylinders, that is, micro-structured bodies, made of a periodic and specifically designed three-dimensional assembly of beams. Derivation is performed in the framework of the direct $1 \mathrm{D}$ approach, while the constitutive law is determined by a homogenization procedure based on an energy equivalence between a cell of the periodic model and a segment of the solid beam. Warping of the cross-section, caused by shear and torsion, is approximatively taken into account by the concept of a shear factor, namely, a corrective factor for the constitutive coefficients of the equivalent beam. The inertial properties of the Timoshenko model are analytically identified under the hypothesis, and the masses are lumped at the joints. Linear static and dynamic responses of some micro-structured beams, taken as case studies, are analyzed, and a comparison between the results given by the Timoshenko model and those obtained by Finite-Element analyses on 3D frames is made. In this framework, the effectiveness of the equivalent model and its limits of applicability are highlighted.
\end{abstract}

Keywords: equivalent beam model; micro-structured beam; homogenization procedure

\section{Introduction}

Recent advances in 3D printing techniques and associated micro-fabrication technologies have made (periodic) micro-structured systems and architected the material object of study in several fields of engineering, ranging from aerospace to biomechanics [1]. Interest in research is particularly focused on some peculiar characteristics of such objects, far exceeding those of conventional bulk materials, like a very low lightweight-stiffness ratio, accompanied with significant mechanical properties, such as resilience and impact resistance, which usually allow for important energy absorption capability, see for example, [2,3], also through micro-scale buckling phenomena. A unit-cell of such micro-structured bodies, whose characteristic dimension is of the order of some millimeters, consists of a lattice of fibers, which interacts at the internal connections. The intrinsic extensional, shear, flexural, and torsional stiffnesses of the fibers, together with the topology of the lattice, is able to confer to the resulting structure, which is the above-mentioned properties at the macro-scale.

Homogenization techniques are very useful in modeling periodic structures as equivalent continua (e.g., micropolar continua [4,5]), such as in the case of lattice structures [6,7], masonry walls [8], lattice beams and plates [9], reticulated structures [10], and pantographic structures [11] (the reader is also referred to the review papers [12-14]), as well in dealing with buckling problems in pantographs $[15,16]$ and micro-structured plates $[17,18]$. Several methods have accordingly been developed in the literature, 
that can be roughly classified into three main categories: (i) heuristic approaches (see, e.g., [19-21]), grounded on an a priori assumption of the homogenized model, without formulating the relations between the micro and macro quantities; (ii) asymptotic homogenization approaches (see, e.g., [22-24]), in which the equivalent continuum is rigorously derived; and (iii) mixed approaches (see, e.g., [25]), in which the continuum model is heuristically predicted and, in addition, the micro-macro relationships are analytically determined on the ground of energy balances.

The mixed up-scaling technique, adopted in the present work, has also been successfully applied to model tower buildings and multi-storey frames as generalized (continuous) beams in [26-35], to describe their overall mechanical behavior in statics [28,30,34,35], dynamics [28,33,35], buckling $[29,32]$, and aeroelasticity $[26,27,31]$ with a lower computational effort, with respect to, for example, Finite-Element models of the correspondent fine structures. These beam-like structures have been macroscopically modeled as shear-shear-torsional beams, that is, where macro-bending has been neglected, or Timoshenko beams, by identifying the floors and the columns of the periodic frames, with the cross-sections and longitudinal fibers of the continuous beam, respectively. In a subset of them [26-30,32,33], identification has been carried out by assuming the floor could behave as a rigid body, both in its plane and out-of-plane; whereas in $[25,34,35]$, this hypothesis has been partially removed and out-of-plane deformability of the floor has been taken into account on an energy average ground. In particular, in [26,27], an equivalent shear-shear-torsional beam model embedded in a 3D-space has been developed to describe the nonlinear aeroelastic behavior of tower buildings. In [30], a rational justification of the main assumptions, heuristically adopted in [27], is provided. In this framework, the limits of applicability of the model have been discussed in terms of nonlinear static response of some buildings. In [33], under a small displacements assumption, the same model was adopted to analyze linear free and forced dynamics of multi-storey buildings, for which both external and internal (proportional) damping forms have been considered, thus allowing a discussion of the damping features of the shear beam model. In [28], a Timoshenko equivalent beam model, embedded in a 3D-space, was developed to also account for macro-bending of multi-storey buildings in linear statics and dynamics. In $[29,32]$, a generalization of this latter model was provided for buckling analyses, including for the geometric effects both in the equilibrium equations and in the constitutive law-in this context, the effect of uniform compression ([29]), as well as that of non-uniform compression and the interactions with elastic soil ([32]), are discussed. In [34], by limiting the analysis to planar frames, it has been shown that it is possible to consider the out-of plane true flexibility of the cross-section, even when rigid cross-section 1D (unwarpable) models, such as the shear and the Timoshenko ones, are heuristically adopted. Indeed, corrective factors have been analytically derived in [34] for the case of geometrically regular planar frames, to suitably reduce the shear and flexural stiffnesses of the macro-model by accounting on (energy) averages for the neglected warping. This analysis has been extended in [35], where an energy-based numerical algorithm, sided on a Finite-Element (FE) analysis of a single cell, has been again applied to planar frames, in order to detect the constitutive properties of the equivalent beam model. Finally, in [25], a review of these works was presented, together with a systematization of the homogenization procedure there adopted.

In this paper, the analysis so far carried out in $[25,34,35]$ is extended to the spatial behavior of micro-structured beams, here referred to as grid beams, which are namely cylinders made of a cubic micro-structure [36], embedded in a 3D space, consisting of a pattern of three orders of orthogonal micro-beams periodically arranged. In particular, an equivalent 1D Timoshenko beam model is formulated through a direct approach. Moreover, the out-of-plane deformability of the cross-section, induced by shear and torsion, is approximatively taken into account by introducing corrective factors for the constitutive coefficients of the equivalent beam model. Attention is focused on statics and free dynamics of such structures. It is worth noticing that the main goal of the paper relies on the interpretation of the mechanical behaviour of such a complex micro-structured and three-dimensional object, and not on the algorithmic formulation. In particular, it is of interest to prove 
that the observation on a large scale of the mechanics of a periodic system can be pursued (with a good approximation) by using the classical tools of Continuum Mechanics.

The paper is organized as follows. In Section 2 the equations of the Timoshenko beam model, embedded in a 3D space, are recalled. In Section 3 the identification procedure is described. In Section 4, comparisons between results provided by Finite-Element models of grid beams and the equivalent Timoshenko model, for which exact solutions are viable, are developed. In Section 5, some conclusions and perspectives of this work are drawn.

\section{Model}

The grid beam consists of a grid cylinder, that is, in a 3D frame, made of a periodic and specifically designed three-dimensional assembly of equal micro-beams. It is made of micro-beams, here referred to as fibers, forming a periodic cubic micro-structure, of side $h$ (Figure 1a), whose pattern is repeated $n_{x}=n$ times in $x$-direction. Two families of fibers are detected: (i) those parallel to the beam $x$-axis, referred to as longitudinal fibers, which are $n_{y}$ in $y$-direction and $n_{z}$ in $z$-direction; (ii) those lying in the $n+1$ planes orthogonal to the axis-line, referred to as transverse fibers, parallel to the $(y, z)$-plane. These latter are arranged in a square orthogonal pattern that is reinforced through bracing diagonal elements, which guarantee the indeformability of the cylinder in the $(y, z)$-plane. It is assumed that the $(x, y)$ - and $(x, z)$-plane are of symmetry for the grid beam, and the origin of the coordinate system is at the lower end $A$ (see Figure 1). All the fibers are modeled as Timoshenko micro-beams, whose cross-section properties are taken as equal in the two principal inertia planes, where square, circular, and ring cross-sections, or analogous, are considered. They have axial, shear, bending, and torsional stiffnesses $E A, G A^{*}, E I$, and $G J$, respectively.

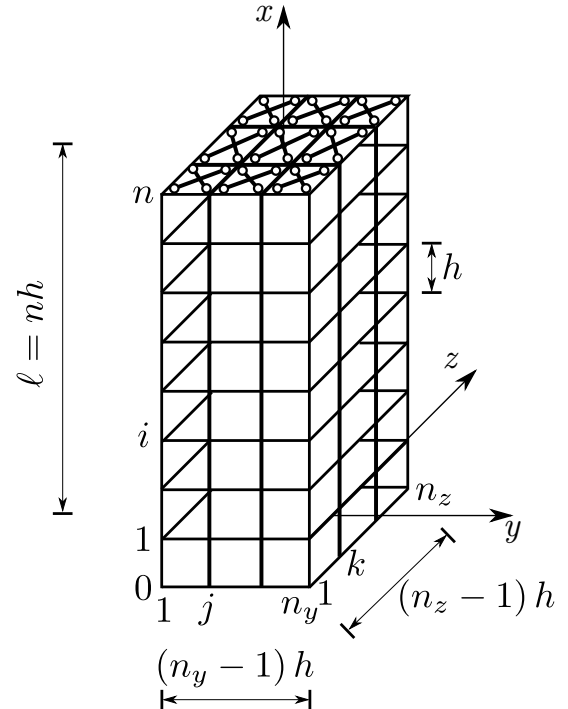

(a)

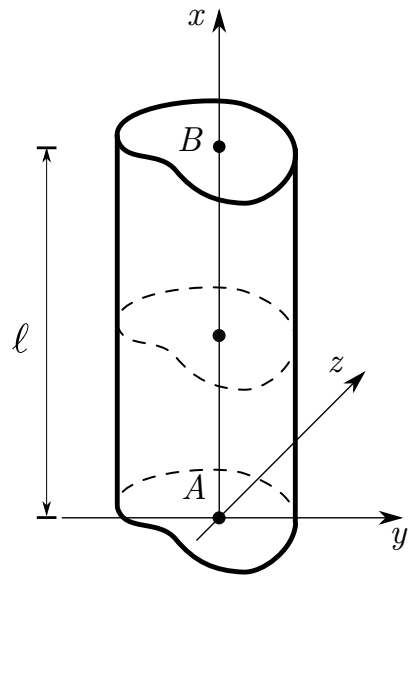

(b)

Figure 1. Study object: (a) grid beam; (b) equivalent beam model.

An internally unconstrained model of beam, embedded in a 3D space, see, for example, [37-42], is formulated (Figure 1b). The beam is made of material points, equipped with orientation, which accounts for the attitude of the cross-section. It is referred to as a material abscissa $x \in$ 
$[0, \ell]$, spanning the interval $(A, B)$ of total length $\ell:=n h$. The following strain-displacement relationships hold:

$$
\begin{aligned}
\varepsilon & =u^{\prime}, \\
\gamma_{y} & =v^{\prime}-\theta_{z} \\
\gamma_{z} & =w^{\prime}+\theta_{y} \\
\kappa_{x} & =\theta_{x}^{\prime} \\
\kappa_{y} & =\theta_{y}^{\prime} \\
\kappa_{z} & =\theta_{z}^{\prime}
\end{aligned}
$$

which link the longitudinal displacement $u(x, t)$, the transverse displacements $v(x, t), w(x, t)$, the in $y$-direction and $z$-direction, respectively, and the rotations of the cross-section $\theta_{v}(x, t)$, with respect to $v$-axis $(v=x, y, z)$, to the elongation $\varepsilon(x, t)$, the shear strains $\gamma_{y}(x, t), \gamma_{z}(x, t)$, the torsional curvature $\kappa_{x}$, and the flexural curvatures $\kappa_{y}(x, t), \kappa_{z}(x, t)$; here, $t$ is the time, and a prime denotes space differentiation.

The following geometric boundary conditions are considered in what follows (subscripts $A, B$ denote evaluation at the ends $x=0$ and $x=\ell$, respectively):

- Clamped at $A$ and free at $B: u_{A}=v_{A}=w_{A}=\theta_{x A}=\theta_{y A}=\theta_{z A}=0$;

- Simply-supported and torsionally restrained in $A, B: u_{A}=u_{B}=v_{A}=v_{B}=w_{A}=w_{B}=\theta_{x A}=$ $\theta_{x B}=0$.

The equilibrium is ruled by the following equations:

$$
\begin{aligned}
& N^{\prime}+p_{x}-m \ddot{u}=0, \\
& T_{y}^{\prime}+p_{y}-m \ddot{v}=0, \\
& T_{z}^{\prime}+p_{z}-m \ddot{w}=0, \\
& M_{x}^{\prime}+c_{x}-I_{x} \ddot{\theta_{x}}=0, \\
& M_{y}^{\prime}-T_{z}+c_{y}-I_{y} \ddot{\theta_{y}}=0, \\
& M_{z}^{\prime}+T_{y}+c_{z}-I_{z} \ddot{\theta}_{z}=0,
\end{aligned}
$$

where $N(x, t), T_{y}(x, t)$ and $T_{z}(x, t)$ are the axial and shear internal forces, respectively, $M_{x}(x, t)$ is the torsional moment, $M_{y}(x, t)$ and $M_{z}(x, t)$ are the bending moments, $p_{v}(x, t), c_{v}(x, t)$ are the external forces and couple per unit-length $(v=x, y, z)$. Moreover, $m$ and $I_{v}$ are the mass and second inertia moments per unit-length, evaluated with respect to the centroid $(v=x, y, z)$, and a dot denotes time-differentiation.

When no external load and no lumped masses are present at the ends of the beam, the mechanical boundary conditions read:

- $\quad$ Clamped at $A$ and free at $B: N_{B}=T_{y B}=T_{z B}=M_{x B}=M_{y B}=M_{z B}=0$;

- Simply supported and torsionally restrained in $A, B: M_{y A}=M_{y B}=M_{z A}=M_{z B}=0$.

It is assumed that the beam material is hyperelastic, so that the existence of a strain energy function, quadratic in the strains, is postulated, namely:

$$
\phi=\frac{1}{2}\left(c_{11} \varepsilon^{2}+c_{22} \gamma_{y}^{2}+c_{33} \gamma_{z}^{2}+c_{44} \kappa_{x}^{2}+c_{55} \kappa_{y}^{2}+c_{66} \kappa_{z}^{2}\right),
$$


where the elastic constants $c_{i i}(i=1, \ldots, 6)$ must be identified according to the micro-structure of the grid beam. The constitutive law follows from the Green law as:

$$
\left(\begin{array}{c}
N \\
T_{y} \\
T_{z} \\
M_{x} \\
M_{y} \\
M_{z}
\end{array}\right)=\left(\begin{array}{cccccc}
c_{11} & 0 & 0 & 0 & 0 & 0 \\
& c_{22} & 0 & 0 & 0 & 0 \\
& & c_{33} & 0 & 0 & 0 \\
& & & c_{44} & 0 & 0 \\
& & & & c_{55} & 0 \\
\text { SYM } & & & & & c_{66}
\end{array}\right)\left(\begin{array}{c}
\varepsilon \\
\gamma_{y} \\
\gamma_{z} \\
\kappa_{x} \\
\kappa_{y} \\
\kappa_{z}
\end{array}\right) .
$$

Then, equilibrium (2) is written in terms of displacements, and by using Equations (4) and (1), the elasto-dynamic problem is obtained:

$$
\begin{aligned}
& -\mathbf{M} \ddot{\mathbf{u}}+\mathbf{K}_{2} \mathbf{u}^{\prime \prime}+\mathbf{K}_{1} \mathbf{u}^{\prime}+\mathbf{K}_{0} \mathbf{u}+\mathbf{p}=\mathbf{0}, \\
& \mathbf{A}_{1} \mathbf{u}_{A}^{\prime}+\mathbf{A}_{0} \mathbf{u}_{A}=\mathbf{0}, \\
& \mathbf{B}_{1} \mathbf{u}_{B}^{\prime}+\mathbf{B}_{0} \mathbf{u}_{B}=\mathbf{0} .
\end{aligned}
$$

Equation (5)-a is a system of partial differential equations, sided by geometric and mechanical boundary conditions at $A$ and $B$, Equation (5)-b,c, and by initial conditions, prescribing that the system is initially at rest (not reported here). Moreover,

$$
\begin{array}{rlrl}
\mathbf{u}:=\left(u, v, w, \theta_{x}, \theta_{y}, \theta_{z}\right)^{T}, & \mathbf{p}:=\left(p_{x}, p_{y}, p_{z}, c_{x}, c_{y}, c_{z}\right)^{T}, \\
\mathbf{M}:=\operatorname{diag}\left(m, m, m, I_{x}, I_{y}, I_{z}\right), & \mathbf{K}_{2}:=\operatorname{diag}\left(c_{11}, c_{22}, c_{33}, c_{44}, c_{55}, c_{66}\right), \\
\mathbf{K}_{1}:=\left(\begin{array}{cccccc}
0 & 0 & 0 & 0 & 0 \\
& 0 & 0 & 0 & 0 & -c_{22} \\
& 0 & 0 & c_{33} & 0 \\
\text { SKW } & & 0 & 0 & 0 \\
0 & & & & 0
\end{array}\right), \quad \mathbf{K}_{0}:=\left(\begin{array}{ccccc}
0 & 0 & 0 & 0 & 0 \\
0 & 0 & 0 & 0 & 0 \\
& 0 & 0 & 0 & 0 \\
& & 0 & 0 & 0 \\
& & & -c_{33} & 0 \\
\text { SYM } & & & & -c_{22}
\end{array}\right),
\end{array}
$$

where $\mathbf{M}$ is the mass matrix, $\mathbf{K}_{i}(i=0,1,2)$ are symmetric or skew-symmetric stiffness matrices, and $\mathbf{u}, \mathbf{p}$ are displacement and load vectors, respectively. Boundary operators, involved in Equation (5)-b,c, are defined as:

- Clamped at $A$ and free at $B$ :

$$
\begin{aligned}
\mathbf{A}_{1}:=\mathbf{0}, & \mathbf{A}_{0}:=\mathbf{I}, \\
\mathbf{B}_{1}:=\mathbf{K}_{2}, \quad \mathbf{B}_{0}:= & \left(\begin{array}{cccccc}
0 & 0 & 0 & 0 & 0 & 0 \\
0 & 0 & 0 & 0 & 0 & -c_{22} \\
0 & 0 & 0 & 0 & c_{33} & 0 \\
0 & 0 & 0 & 0 & 0 & 0 \\
0 & 0 & 0 & 0 & 0 & 0 \\
0 & 0 & 0 & 0 & 0 & 0
\end{array}\right) ;
\end{aligned}
$$

- Simply-supported and torsionally restrained in $A, B$ :

$$
\mathbf{A}_{1}=\mathbf{B}_{1}:=\operatorname{diag}\left(0,0,0,0, c_{55}, c_{66}\right), \quad \mathbf{A}_{0}=\mathbf{B}_{0}:=\operatorname{diag}(1,1,1,1,0,0) .
$$

It is worth noticing that in the static case, that is, when inertial effects are disregarded, the elastic problem (5) consists in a system of ordinary differential equations and boundary conditions in the unknown displacement field. They can be easily solved in closed-form due to the uncoupled 
constitutive law, entailing that the equations of the equivalent model are the classical (partially uncoupled) equations of the Timoshenko beam model [43]. Moreover, the free dynamics problem associated with Equation (5) also admits a closed-form solution, since the mass matrix is diagonal; see [43-45].

\section{Elastic and Inertial Constant Identifications}

\subsection{Identification Algorithm for the Elastic Constants}

The identification of the elastic constants follows the lines of [25], according to which a suitable energy equivalence between the (fine) 3D grid beam model and the (coarse) 1D Timoshenko beam model is enforced (time is understood in what follows). The analysis here presented extends to the 3D case, the results of [25], where a planar grid beam was considered.

The first step of the identification consists in defining a discrete map, which links the displacements of the fine model to that of the coarse one, at the selected abscissas $x_{i}:=$ ih $(i=0,1, \ldots, n), h$ being the period of the grid beam. At each $x_{i}$, the fine model possesses a cross-section $\mathcal{D}_{i}$ occupying a domain of the $(y, z)$-plane, made of the nodal points of the $(y, z)$-fibers; two adjacent cross-sections $\mathcal{D}_{i}, \mathcal{D}_{i+1}$ bound a cell. In order to define the displacement map, a kinematic constraint is enforced-the sampled cross-section $\mathcal{D}_{i}$ remains plane, while the displacements inside the cells are unconstrained. The rigid displacement of $\mathcal{D}_{i}$ (the tilde denoting, from now on, a quantity relevant to the $3 \mathrm{D}$ body), can be written as:

$$
\left(\begin{array}{c}
\tilde{u}\left(x_{i}, y, z\right) \\
\tilde{v}\left(x_{i}, y, z\right) \\
\tilde{w}\left(x_{i}, y, z\right)
\end{array}\right)=\left(\begin{array}{c}
u\left(x_{i}\right) \\
v\left(x_{i}\right) \\
w\left(x_{i}\right)
\end{array}\right)+\left[\begin{array}{ccc}
0 & -\theta_{z}\left(x_{i}\right) & \theta_{y}\left(x_{i}\right) \\
& 0 & -\theta_{x}\left(x_{i}\right) \\
\mathrm{SKW} & & 0
\end{array}\right]\left(\begin{array}{c}
0 \\
y \\
z
\end{array}\right), \quad \operatorname{in} \mathcal{D}_{i}, i=0,1, \ldots, n .
$$

The second step requires enforcement of the equivalence of the energies stored by a 3D cell and a segment of equal length of the 1D beam, when the two models undergo the same displacements at the ends. This (so-called) cell analysis leads to the evaluation of the averaged energy density of the 3D cell, namely $\tilde{\phi}:=\tilde{U} / h$, with $\tilde{U}$ being the elastic energy of the 3D cell, as a function of the configuration variables of the 1D beam (according to Equation (9)). The energy density of the cell reads:

$$
\tilde{\phi}=\frac{1}{h} \tilde{U}\left(u\left(x_{i}\right), v\left(x_{i}\right), w\left(x_{i}\right), \theta_{v}\left(x_{i}\right), u\left(x_{i+1}\right), v\left(x_{i+1}\right), w\left(x_{i+1}\right), \theta_{v}\left(x_{i+1}\right)\right), \quad v=x, y, z .
$$

The last step of the identification consists in a comparison between the energy densities $\tilde{\phi}$ and $\phi$, in Equations (3) and (10), respectively. To this end, the strain-displacement relationships (1) must be integrated in the $\left(x_{i}, x_{i+1}\right)$ interval, thus giving a suitable strain test field. By taking the (simplest) constant strain field, the end displacements (rigid motions removed) read:

$$
\begin{array}{rlrl}
u\left(x_{i}\right) & =0, & u\left(x_{i+1}\right)=\varepsilon h, \\
v\left(x_{i}\right)=0, & v\left(x_{i+1}\right)=\gamma_{y} h+\frac{1}{2} \kappa_{z} h^{2}, \\
w\left(x_{i}\right)=0, & w\left(x_{i+1}\right)=\gamma_{z} h-\frac{1}{2} \kappa_{y} h^{2}, \\
\theta_{x}\left(x_{i}\right)=0, & \theta_{x}\left(x_{i+1}\right)=\kappa_{x} h, \\
\theta_{y}\left(x_{i}\right)=0, & \theta_{y}\left(x_{i+1}\right)=\kappa_{y} h, \\
\theta_{z}\left(x_{i}\right)=0, & \theta_{z}\left(x_{i+1}\right)=\kappa_{z} h .
\end{array}
$$

Once Equation (11) is substituted in Equation (10), by the equality $\phi=\tilde{\phi} \quad \forall\left(\varepsilon, \gamma_{y}, \gamma_{z}, \kappa_{x}, \kappa_{y}, \kappa_{z}\right)$, the elastic constants $\mathbf{C}:=\operatorname{diag}\left(c_{i i}\right), i=1, \ldots, 6$ follow.

It is important to remark that the cell's boundary displacements, defined in Equation (11), can be read as the result of a superposition of six independent deformation modes, namely: 
- An extensional mode (EX), in which $\mathcal{D}_{i+1}$ translates axially;

- Two shear modes $\left(\mathrm{SH}_{y}, \mathrm{SH}_{z}\right)$, in which $\mathcal{D}_{i+1}$ translates transversely along the $y$ - and $z$-directions, respectively;

- two flexural modes $\left(\mathrm{FL}_{y}, \mathrm{FL}_{z}\right)$, in which $\mathcal{D}_{i+1}$ rotates around the $y$ - and $z$-axes, respectively, and translates transversely along the $z$ - and $y$-axes;

- $\quad$ A torsional mode (TO), in which $\mathcal{D}_{i+1}$ rotates around the $x$ - axis.

Moreover, it is worth noticing that the evaluation of the cell's elastic energy is the most difficult step in the identification procedure and, in general, it cannot be pursued in closed form except for few cases, where for example, the fibers are arranged in a simple and regular pattern and/or the cross-section is assumed to be rigid (see $[28,32,34])$. On the contrary, a numerical identification procedure, such as that based on a Finite Element analysis, is always viable, as done in [35].

\section{Analytical Identification of Elastic Constants of the Grid Beam}

The illustrated algorithm is applied to the grid beam. A cell made of $n_{y} \times n_{z}$ longitudinal fibers, comprised between two adjacent cross-sections $\mathcal{D}_{i}, \mathcal{D}_{i+1}$, is considered. The fibers belonging to the cross-sections are taken of half of their stiffnesses, and only their nodal points are assumed to remain on the same plane, as displayed in Figure 2. However, the same fibers are free to warp around the plane-this deformation is here referred to as micro-warping.

The previously introduced deformation modes, shown in Figure 2, are then assigned to the cell under the following assumptions:

Hypothesis 1. In the shear modes $\mathrm{SH}_{y}, \mathrm{SH}_{z}$ all nodes rotate of the unknown angles $\varphi_{y}$ and $\varphi_{z}$, respectively (see [34]);

Hypothesis 2. In the flexural modes $\mathrm{FL}_{y}, \mathrm{FL}_{z}$ the effect of micro-warping is neglected, since the associated elastic energy is small with respect to the extension of the longitudinal fibers (see [34]);

Hypothesis 3. In the torsional mode TO the following energy contributions, related to the fibers belonging to the cross-section plane, are neglected:

- That due to their bending in the cross-section plane;

- That due to their torsion.

It is worth noticing that Hypothesis 3 has been numerically validated in what follows on case studies, as it will be shown ahead.

The energy $\tilde{U}_{\mathrm{EX}}$, associated to the EX-mode reads:

$$
\tilde{U}_{\mathrm{EX}}=\frac{1}{2} n_{y} n_{z} E A h \varepsilon^{2},
$$

in which the only contribution is due to the extension of the longitudinal fibers. 


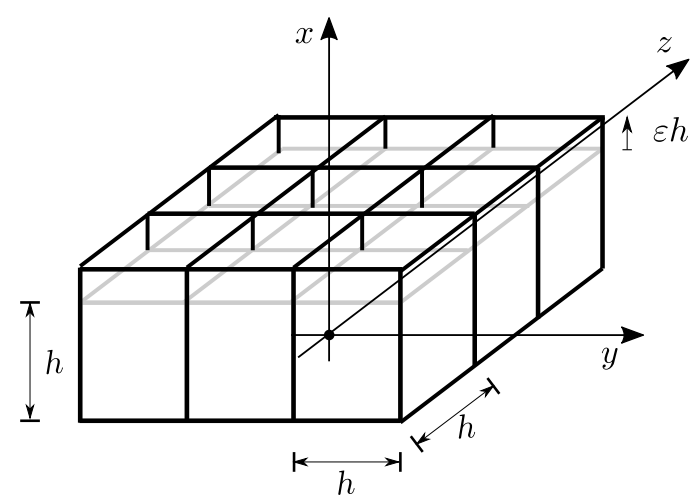

(a)

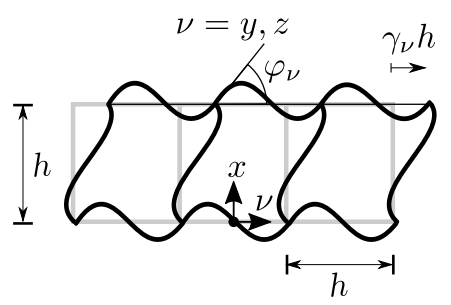

(c)

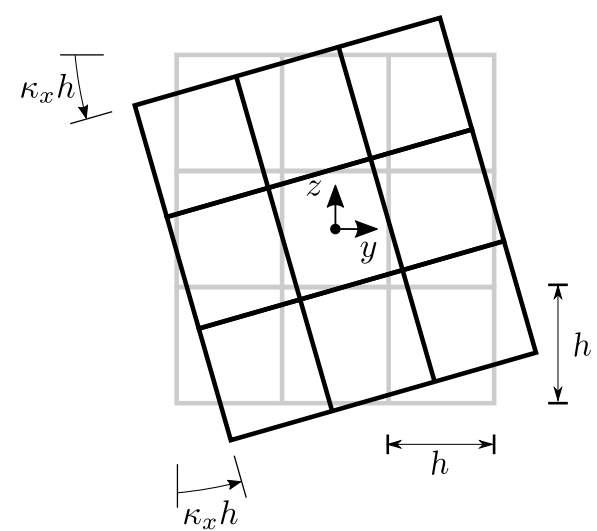

(b)

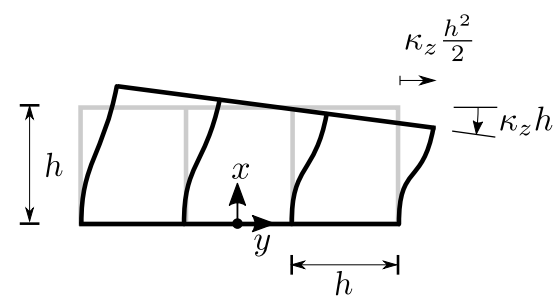

(d)

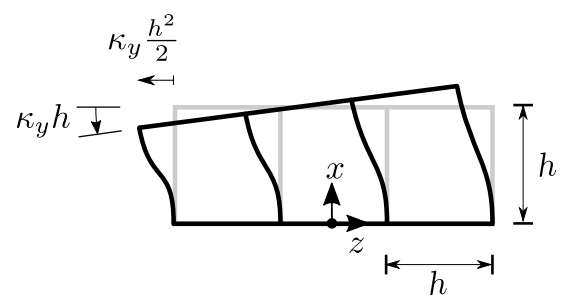

(e)

Figure 2. Single cell of the grid beam, and its deformation modes (bracings not shown): (a) 3D view of the EX mode; (b) top view of the TO mode; (c) lateral view of the $\mathrm{SH}_{v}$ mode, $v=y, z$; (d,e) lateral view of the $\mathrm{FL}_{v}$ mode, $v=y, z$.

The energies $\tilde{U}_{\mathrm{SH}_{y}}, \tilde{U}_{\mathrm{SH}_{z}}$ associated to the correspondent shear modes can be determined via Clapeyron's theorem, that is, as with the deformation work spent by the end-forces of each fiber in the correspondent (assigned) nodal displacements. They read (see [34] for details):

$$
\begin{aligned}
& \tilde{U}_{\mathrm{SH}_{y}}=n_{y} n_{z} \frac{6 E I}{h(1+\alpha)}\left(\gamma_{y}-\varphi_{y}\right)^{2}+\left(n_{y}-1\right) n_{z} \frac{6 E I}{h(1+\alpha)} \varphi_{y^{\prime}}^{2} \\
& \tilde{U}_{\mathrm{SH}_{z}}=n_{y} n_{z} \frac{6 E I}{h(1+\alpha)}\left(\gamma_{z}-\varphi_{z}\right)^{2}+\left(n_{z}-1\right) n_{y} \frac{6 E I}{h(1+\alpha)} \varphi_{z^{\prime}}^{2}
\end{aligned}
$$

where the two contributions on the right-end sides of Equation (13) are related to bending of the longitudinal fibers and micro-warping of the transverse fibers, respectively. Moreover, $\alpha:=$ $12 E I /\left(G A^{*} h^{2}\right)$ is a nondimensional stiffness ratio. By requiring $\frac{\partial \tilde{U}_{\mathrm{SH}_{y}}}{\partial \varphi_{y}}=0, \frac{\partial \tilde{U}_{\mathrm{SH}_{z}}}{\partial \varphi_{z}}=0$ the unknowns $\varphi_{y}$ and $\varphi_{z}$ are eliminated, and Equation (13) reads: 


$$
\begin{aligned}
\tilde{U}_{\mathrm{SH}_{y}} & =\frac{1}{2} \chi_{y} n_{y} n_{z} \frac{12 E I}{(1+\alpha) h} \gamma_{y}^{2}, \\
\tilde{U}_{\mathrm{SH}_{z}} & =\frac{1}{2} \chi_{z} n_{y} n_{z} \frac{12 E I}{(1+\alpha) h} \gamma_{z}^{2},
\end{aligned}
$$

where:

$$
\chi_{y}:=\frac{n_{y}-1}{2 n_{y}-1}, \quad \chi_{z}:=\frac{n_{z}-1}{2 n_{z}-1},
$$

are nondimensional quantities, here referred to as shear factors, which, as shown in [34], account for the micro-warping of the fibers (i.e., a warping which leaves the joints aligned) of the fine model in the same way they account for warping of the solid cross-section in the Timoshenko beam model.

The energies $\tilde{U}_{\mathrm{FL}_{y}}, \tilde{U}_{\mathrm{FL}_{z}}$ associated to the correspondent flexural modes are:

$$
\begin{aligned}
& \tilde{U}_{\mathrm{FL}_{y}}=\frac{1}{2} n_{y}\left(E I h n_{z}+E A h \sum_{j=1}^{n_{z}} z_{j}^{2}\right) \kappa_{y}^{2} \\
& \tilde{U}_{\mathrm{FL}_{z}}=\frac{1}{2} n_{z}\left(E I h n_{y}+E A h \sum_{k=1}^{n_{y}} y_{k}^{2}\right) \kappa_{z}^{2},
\end{aligned}
$$

where the two contributions on the right-end sides of Equation (16) accounts for bending and extension of the longitudinal fibers. Here, according to that discussed in [34], micro-warping of $y$ - and $z$-fibers is neglected.

The energy $\tilde{U}_{\mathrm{TO}}$, associated with the torsional mode $\mathrm{TO}$, is the sum of the torsional energies: (i) of its longitudinal fibers, which, according to the Hypothesis 3, experience the same twist angle; and (ii) of the two orthogonal orders of planar frames parallel to $y$ and $z$. Concerning this latter contribution, it is found by evaluating the torsional energy of a single planar frame, undergoing a shear mode, namely $\gamma_{y j}=-\kappa_{x} z_{j}$ (y -parallel) or $\gamma_{z k}=\kappa_{x} y_{k}$ (z-parallel), analogously to what was done to determine Equation (14). Accordingly, $\tilde{U}_{\mathrm{TO}}$ reads:

$$
\tilde{U}_{\mathrm{TO}}=\frac{1}{2}\left[n_{y} n_{z} G J h+\chi_{y} n_{y} \frac{12 E I}{h(1+\alpha)} \sum_{j=1}^{n_{z}} z_{j}^{2}+\chi_{z} n_{z} \frac{12 E I}{h(1+\alpha)} \sum_{k=1}^{n_{y}} y_{k}^{2}\right] \kappa_{x}^{2}
$$

where the terms in the square brackets are the torsional stiffnesses of the longitudinal fibers, and of the frames parallel to the $(x, z)$ - and $(y, z)$-planes, respectively.

Moreover, since $\tilde{U}=\tilde{U}_{\mathrm{EX}}+\tilde{U}_{\mathrm{SH}_{y}}+\tilde{U}_{\mathrm{SH}_{z}}+\tilde{U}_{\mathrm{FL}_{y}}+\tilde{U}_{\mathrm{FL}_{z}}+\tilde{U}_{\mathrm{TO}}$, Equation (10) supplies $\tilde{\phi}$; then, the equality $\phi=\tilde{\phi} \quad \forall\left(\varepsilon, \gamma_{y}, \gamma_{z}, \kappa_{x}, \kappa_{y}, \kappa_{z}\right)$ is enforced and the Green law supplies the following elastic constants, Equation (4), namely:

$$
\begin{aligned}
& c_{11}=n_{y} n_{z} E A, \\
& c_{22}=\chi_{y} n_{y} n_{z} \frac{12 E I}{h^{2}(1+\alpha)} \\
& c_{33}=\chi_{z} n_{y} n_{z} \frac{12 E I}{h^{2}(1+\alpha)} \\
& c_{44}=n_{y} n_{z} G J+\chi_{y} n_{y} \frac{12 E I}{h^{2}(1+\alpha)} \sum_{j=1}^{n_{z}} z_{j}^{2}+\chi_{z} n_{z} \frac{12 E I}{h^{2}(1+\alpha)} \sum_{k=1}^{n_{y}} y_{k}^{2} \\
& c_{55}=n_{y}\left(E I n_{z}+E A \sum_{j=1}^{n_{z}} z_{j}^{2}\right), \\
& c_{66}=n_{z}\left(E I n_{y}+E A \sum_{k=1}^{n_{y}} y_{k}^{2}\right) .
\end{aligned}
$$


As a final remark, it is important to observe that, since bracing elements are assumed to be hinged, they do not energetically contribute to $\tilde{U}$. Accordingly, their stiffness does not enter in the definition of the elastic constants (18).

\subsection{Identification of the Inertial Properties}

The inertial properties of the grid beam are identified in this section for dynamical analysis. A cell is taken, made of: (i) transverse fibers, belonging to a generic cross-section $\mathcal{D}_{i}$, and (ii) half of the $x$-fibers below it and half above it. The mass of the fibers and that of the bracings is assumed to be lumped at joints. The kinetic energy of the cell, $\tilde{\mathcal{T}}$, is then determined and equated to the kinetic energy $\mathcal{T}$ of a segment of beam of same length $h$, subjected to a rigid field of velocity:

$$
\begin{aligned}
& \tilde{\mathcal{T}}=\frac{1}{2} \sum_{j=1}^{n_{y}} \sum_{k=1}^{n_{z}} M_{j k}\left(\dot{u}_{j k}^{2}+\dot{v}_{j k}^{2}+\dot{w}_{j k}^{2}\right), \\
& \mathcal{T}=\frac{h}{2}\left[m\left(\dot{u}^{2}+\dot{v}^{2}+\dot{w}^{2}\right)+I_{x} \dot{\theta}_{x}^{2}+I_{y} \dot{\theta}_{y}^{2}+I_{z} \dot{\theta}_{z}^{2}\right],
\end{aligned}
$$

where $M_{j k}$ are the lumped masses at the $(j, k)$-joint, of coordinates $\left(y_{j k}, z_{j k}\right)$, and $\dot{u}_{j k}, \dot{v}_{j k}, \dot{w}_{j k}$ the correspondent translational velocities. By using the displacement map (9), it follows that:

$$
\begin{aligned}
& m=\frac{1}{h} \sum_{j=1}^{n_{y}} \sum_{k=1}^{n_{z}} M_{j k}, \\
& I_{x}=\frac{1}{h} \sum_{j=1}^{n_{y}} \sum_{k=1}^{n_{z}} M_{j k}\left(y_{j k}^{2}+z_{j k}^{2}\right), \\
& I_{y}=\frac{1}{h} \sum_{j=1}^{n_{y}} \sum_{k=1}^{n_{z}} M_{j k} z_{j k \prime}^{2} \\
& I_{z}=\frac{1}{h} \sum_{j=1}^{n_{y}} \sum_{k=1}^{n_{z}} M_{j k} y_{j k}^{2} .
\end{aligned}
$$

\section{Numerical Results}

Numerical results are referred to as two grid beams, taken as case studies, made of a thermoplastic polymer, having elastic modulus $E=2180 \mathrm{~N} / \mathrm{mm}^{2}$, Poisson coefficient $v=0$ and mass density $\rho=1040 \mathrm{~kg} / \mathrm{m}^{3}$. The layout of the longitudinal fibers for the two case studies is shown in Figure 3 , namely: in case study I (Figure 3a) a squared (or "compact") layout is considered, while in case study II (Figure 3b) a rectangular (or "thin") layout is taken. All the fibers and bracing elements have equal square cross-sections, of dimensions $1.5 \mathrm{~mm} \times 1.5 \mathrm{~mm}$. The elasto-geometric characteristics of the cross-section are reported in Table 1 ( $A$ is the area, $A^{*}$ the shear area, $I$ the inertia moment, $J$ the torsional inertia). 


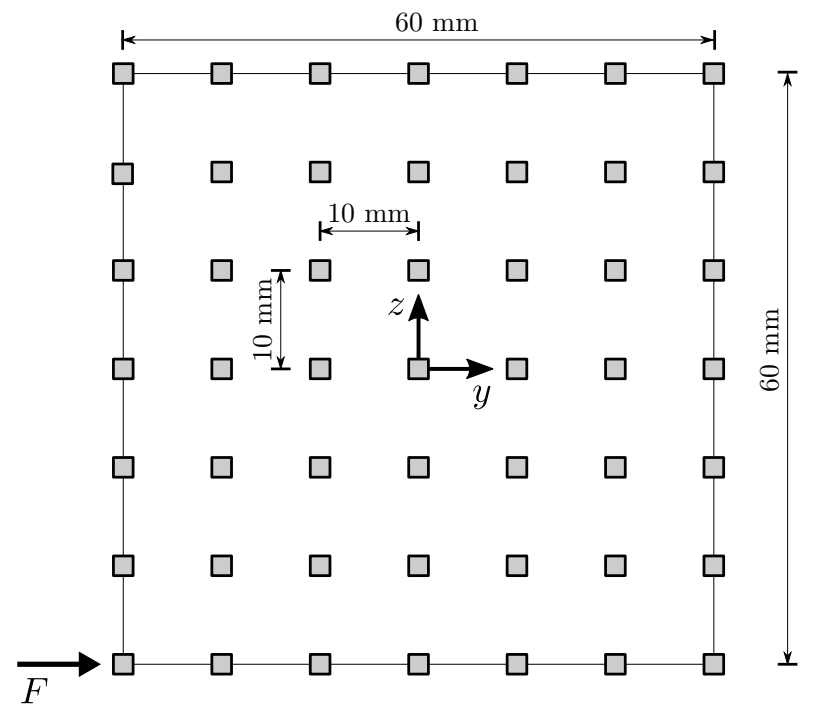

(a)

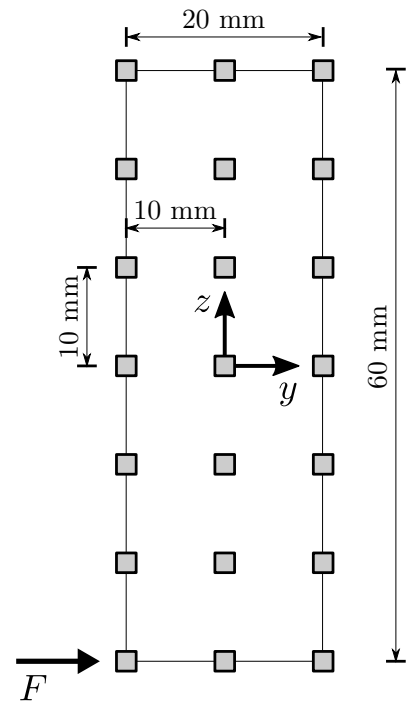

(b)

Figure 3. Grid beam $x$-fibers layout: (a) case study I; (b) case study II.

Table 1. Elasto-geometric fibers' properties for the case studies I and II.

\begin{tabular}{cccc}
\hline$E A$ & $G A^{*}$ & $E I$ & $G J$ \\
{$[\mathbf{N}]$} & {$[\mathbf{N}]$} & {$\left[\mathbf{N} \times \mathbf{m m}^{\mathbf{2}}\right]$} & {$\left[\mathbf{N} \times \mathbf{m m}^{\mathbf{2}}\right]$} \\
\hline 4905 & 2043.75 & 919.69 & 778.06 \\
\hline
\end{tabular}

The considered boundary conditions are: (i) clamped at the ground, case studies denoted with "-a", or (ii) simply supported and torsionally restrained at the ends, case studies denoted with "-b". The number of cells is taken as $n=40$ or $n=100$ and, in all the cases, $h=10 \mathrm{~mm}$. The static loads consist in horizontal point forces, namely, $F=0.5 \mathrm{~N}$ (placed as in Figure 3), applied at each cross-section, except for the free end cross-section of the clamped case, where $F=0.25 \mathrm{~N}$.

Both static response, under lateral loads, and free dynamics characteristics, accounting for self-mass, are of interest. The analytical (exact) solutions relevant to the equivalent beam model are compared with the numerical results obtained by FE models, in which the grid beam is modeled as a 3D frame. Moreover: (i) a Timoshenko beam (exact) finite-element has been adopted to model the fibers of the grid beam; (ii) each fiber, comprised between two nodes of the frame, has not been meshed in static analyses, while in dynamics, it has been divided into three finite-elements.

The elasto-geometric characteristics of the equivalent beam model are reported in Table 2 for each case study. Moreover, the distributed load and couples assigned to the coarse model are $p_{y}=F / h=$ $0.05 \mathrm{~N} / \mathrm{mm}$ and $c_{x}=(F / h) 3 h=1.5 \mathrm{~N}$. In the following Figures, the green dots represent the FE solution, and the continuous blue curves are relevant to the solution of the Timoshenko beam model, respectively, while the displacement unit is $\mathrm{mm}$. 
Table 2. Elasto-geometric and mass coefficients of the equivalent beam model.

\begin{tabular}{cccc}
\hline & & Case Study I & Case Study II \\
\hline$c_{11}$ & {$[\mathrm{~N}]$} & $2.40 \times 10^{5}$ & $1.03 \times 10^{5}$ \\
$c_{22}$ & {$[\mathrm{~N}]$} & $2.37 \times 10^{3}$ & $8.80 \times 10^{2}$ \\
$c_{33}$ & {$[\mathrm{~N}]$} & $2.37 \times 10^{3}$ & $1.01 \times 10^{3}$ \\
$c_{44}$ & {$\left[\mathrm{~N} \times \mathrm{mm}^{2}\right]$} & $1.93 \times 10^{6}$ & $4.36 \times 10^{5}$ \\
$c_{55}$ & {$\left[\mathrm{~N} \times \mathrm{mm}^{2}\right]$} & $9.62 \times 10^{7}$ & $4.12 \times 10^{7}$ \\
$c_{66}$ & {$\left[\mathrm{~N} \times \mathrm{mm}^{2}\right]$} & $9.62 \times 10^{7}$ & $6.89 \times 10^{6}$ \\
$m$ & {$\left[\mathrm{~kg} / \mathrm{mm}^{-4}\right.$} & $5.44 \times 10^{-4}$ & $2.01 \times 10^{-4}$ \\
$I_{x}$ & {$[\mathrm{~kg} \times \mathrm{mm}]$} & $3.78 \times 10^{-1}$ & $8.13 \times 10^{-2}$ \\
$I_{y}$ & {$[\mathrm{~kg} \times \mathrm{mm}]$} & $1.89 \times 10^{-1}$ & $6.99 \times 10^{-2}$ \\
$I_{z}$ & {$[\mathrm{~kg} \times \mathrm{mm}]$} & $1.89 \times 10^{-1}$ & $1.15 \times 10^{-2}$ \\
\hline
\end{tabular}

\subsection{Statics}

The static analysis here developed leads to a comparison of the fine and coarse models in terms of: (i) lateral displacement $v(x)$ and torsional angle $\theta_{x}(x)$, and (ii) vertical displacements of the grid's cross-sections at selected abscissa $x=\bar{x}$ and $z=0$, namely, $\tilde{u}(\bar{x}, y, 0)$. The aim of this analysis is to check the effectiveness of the Timoshenko beam model in reproducing the static behavior of the grid beam.

A very good accordance is found in cases I-a, irrespectively from the number of cells, that is, $n=40$ (Figure 4 ) and $n=100$ (Figure 5). In the first case, the deformed shape of the equivalent model resembles that of a shear-deformable beam, while in the second case, it resembles that of a slender (Euler) beam. In the former case, the very good agreement between the coarse and fine models is also shown in Figure 6 by comparing their deformed shapes (displacements are amplified by a factor equal to 10 in the Figure). Here, only the joints of the fine model (green dots) are represented, while the blue lines are related to the current configuration of the equivalent model, whose displacements are evaluated by using Equation (9). In Figure 6a, the lateral view at $z=0$ is displayed and some of the cross-sections of the Timoshenko beam, at sampled abscissas $x_{i}$, are shown to compare the two models. Figure $6 \mathrm{~b}, \mathrm{c}$ show two top views of the same deformed shape, at $x=\ell / 2$ and $x=\ell$, respectively, and only the edges of the cross-section of the coarse model are represented for comparison. The order of magnitude of the percentage error, evaluated at $x=\ell$, is about: $4 \%$ for $v(x)$ and $5 \%$ for $\theta_{x}(x)$, when $n=40 ; 0.5 \%$ for $v(x)$ and $7 \%$ for $\theta_{x}(x)$, when $n=100$. In all the cases, the vertical displacements, given by the FE analysis, at different levels, are very well-described by the Timoshenko beam, by remembering the fact that the coarse model is a rigid cross-section model and, therefore, it furnishes the mean-value of the local rotation of the deformable sections of the grid beam (see Figures $4 \mathrm{~b}$ and $5 b$ ), which, indeed, also undergo macro-warping (i.e., misalignment of the joints). 

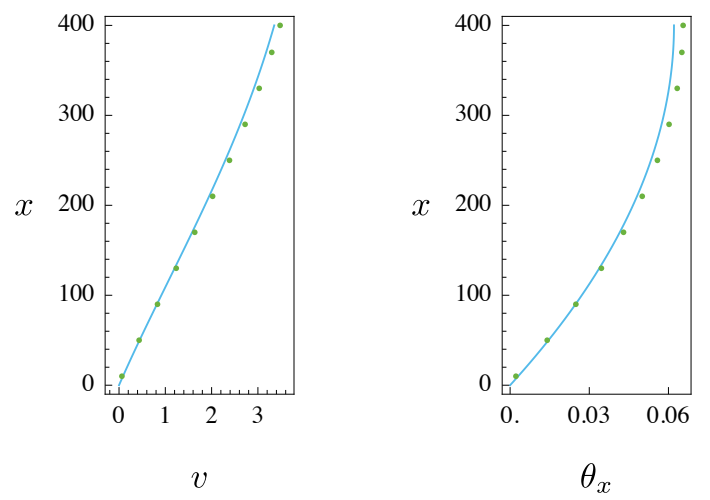

(a)
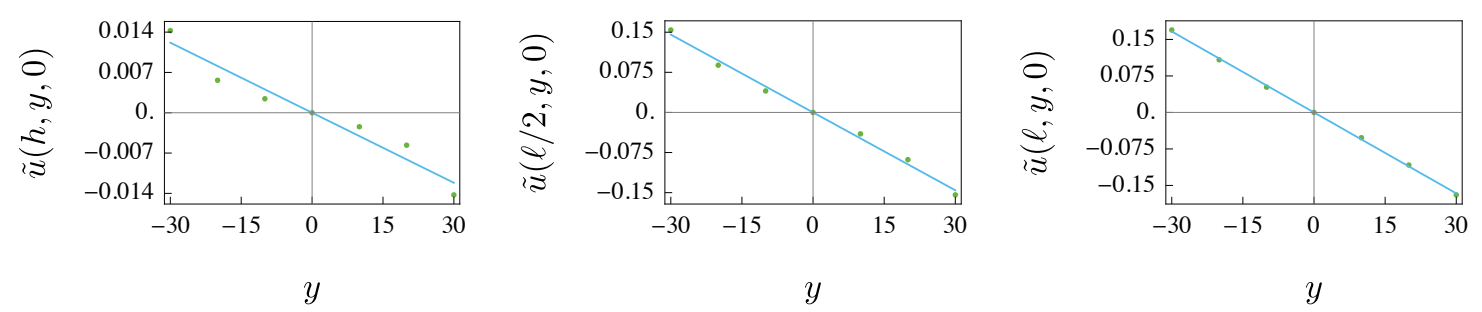

(b)

Figure 4. Static response of the equivalent model vs. the discrete FE model, for case study I-a and $n=40$ : (a) lateral and torsional displacements; (b) vertical displacements of the cross-section joints at $x=h, \ell / 2, \ell$, respectively. Green dots: discrete FE solution. Continuous blue line: homogenized beam model.
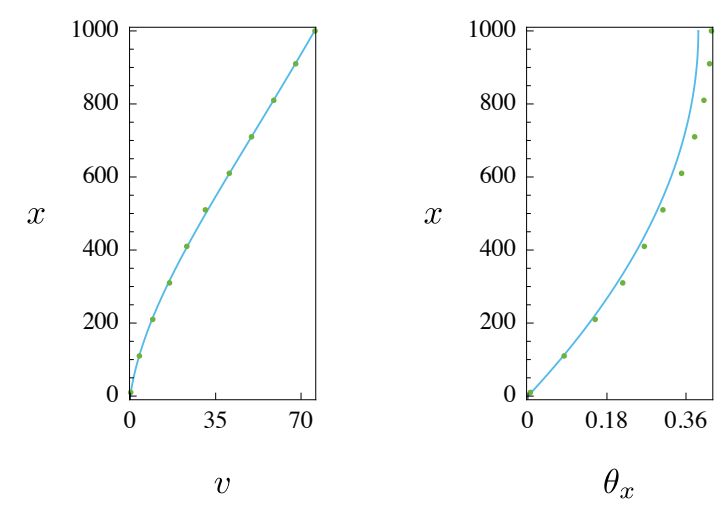

(a)
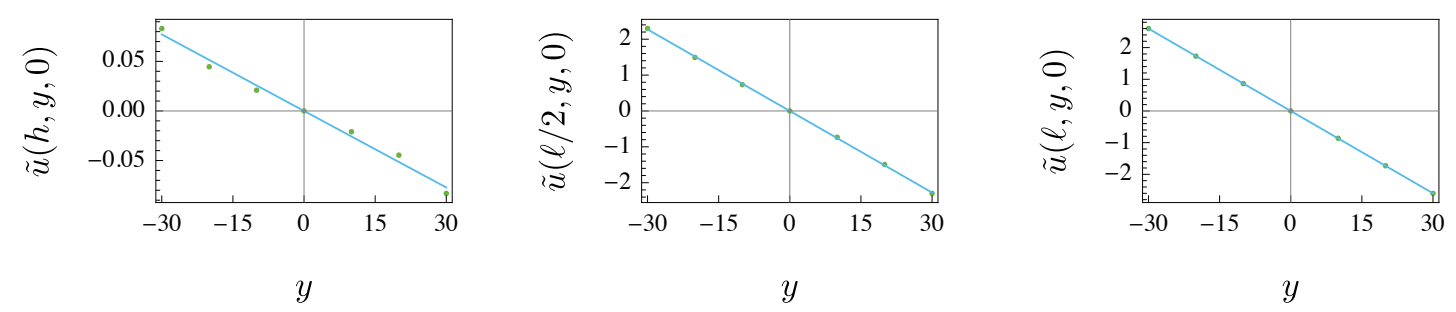

(b)

Figure 5. Static response of the equivalent model vs. the discrete FE model, for case study I-a and $n=100$ : (a) lateral and torsional displacements; (b) vertical displacements of the cross-section joints at $x=h, \ell / 2, \ell$, respectively. Green dots: discrete FE solution. Continuous blue line: homogenized beam model. 


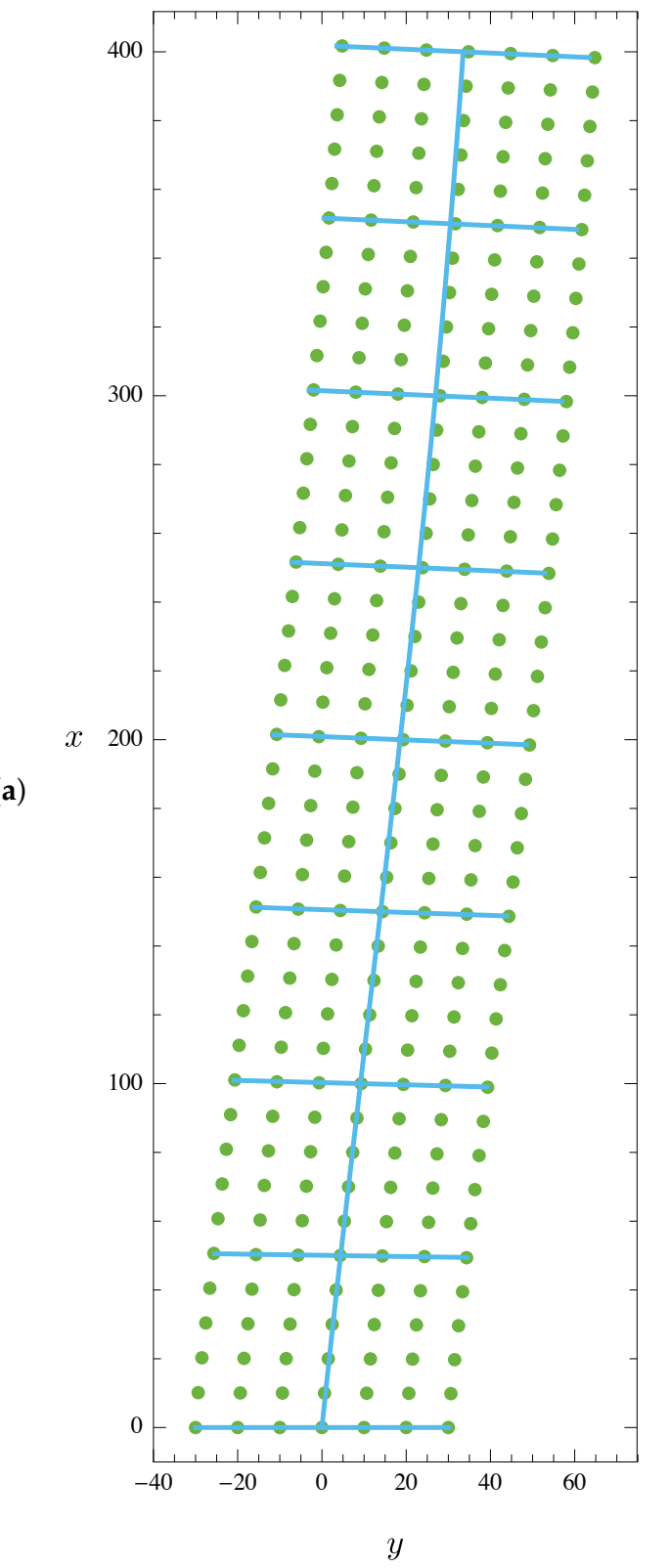

(b)

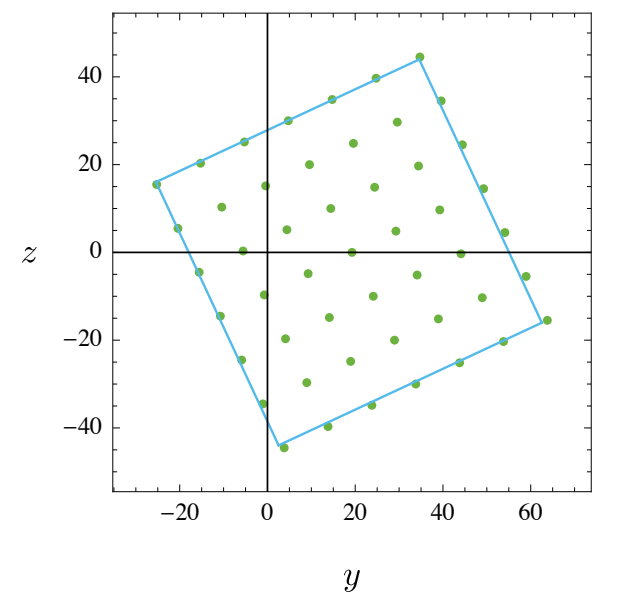

(c)

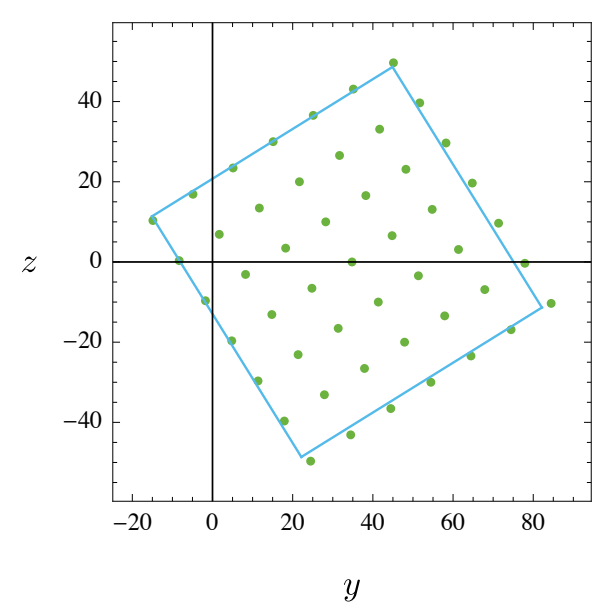

Figure 6. Deformed shape of the equivalent model vs. the discrete FE model, for case study I-a and $n=40$ : (a) lateral view at $z=0 ;(\mathbf{b}, \mathbf{c})$ top views at $x=\ell / 2$ and $x=\ell$, respectively. Green dots: discrete FE solution. Continuous blue line: homogenized beam model. Amplification factor for displacements in the Figure is taken as equal to 10.

The same very good accordance is also found in case I-b, that is, for different boundary conditions, as apparent in Figure 7. Here, the order of magnitude of the percentage error at $x=\ell / 2$ is about $2 \%$ for both $v(x)$ and $\theta_{x}(x)$. In contrast, some important quantitative differences occur for the torsional displacement in case II-a, when $n=40$ (see Figure 8): indeed, here the error at $x=\ell$ is about $0.5 \%$ for $v(x)$ and $32 \%$ for $\theta_{x}(x)$. This occurrence is attributed to the fact that, in case study II, the thinner cross-sections of the grid beam are much more prone to warping, with respect to the (ticker) ones of case I, where the fibers lying in a cross-section plane constitute a more stiff lattice. Therefore, significant macro-warping is experienced from the grid beam in case study II, which cannot be well-captured by a rigid cross-section equivalent model; on the contrary, to account for this effect, more refined beam theories are needed to be able to correctly describe the deformation modes of the cross-sections, as with, for example, the Generalized Beam Theory (see, e.g., [46-48]). 


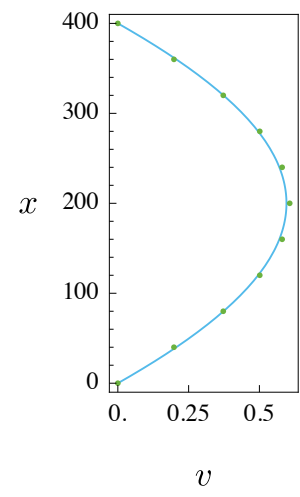

(a)

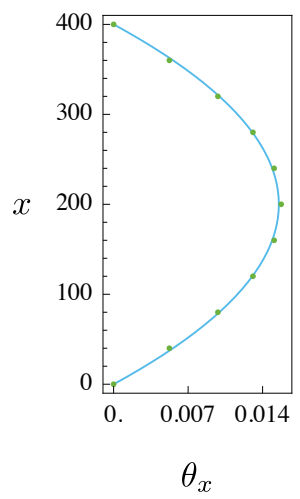

(b)

Figure 7. Static response of the equivalent model vs. discrete FE model, for case study I-b and $n=40$ : (a) lateral displacement; (b) torsional displacement. Green dots: discrete FE solution. Continuous blue line: homogenized beam model.

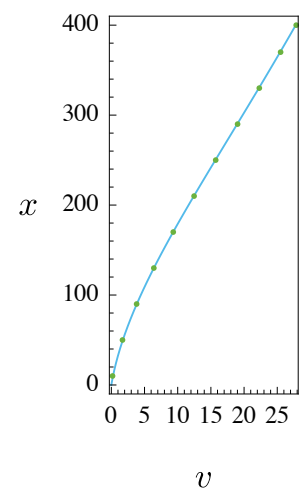

(a)

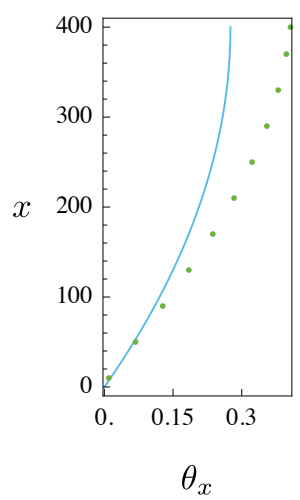

(b)

Figure 8. Static response of the equivalent model vs. discrete FE model, for case study II-a and $n=40$ : (a) lateral displacement; (b) torsional displacement. Green dots: discrete FE solution. Continuous blue line: homogenized beam model.

As proof of what conjectured, a numerical evaluation of the cell's energy, sided on a Finite-Element analysis, has been carried out in cases study I and II, by extending to the 3D case what was done in [35] for a planar cell, namely: (i) the nodal displacements at the boundary joints, compatible with the deformation modes, have been assigned; (ii) the reactive forces at the same joints have been evaluated; and (iii) the elastic energy has been computed via the Clapeyron Theorem. In particular, the cell's energy has been here evaluated by assigning (i) the TO mode, and (ii) an axially-unrestrained version of the TO mode (au-TO), where the kinematic constraint enforcing planarity of the cross-sections $\mathcal{D}_{i}$, is removed and warping is allowed. It was found that:

- case I: $c_{44}=1.90 \times 10^{6} \mathrm{~N} \times \mathrm{mm}^{2}$ (TO mode) and $c_{44}=1.76 \times 10^{6} \mathrm{~N} \times \mathrm{mm}^{2}$ (au-TO mode);

- $\quad$ case II: $c_{44}=4.27 \times 10^{5} \mathrm{~N} \times \mathrm{mm}^{2}$ (TO mode) and $c_{44}=2.36 \times 10^{5} \mathrm{~N} \times \mathrm{mm}^{2}$ (au-TO mode).

It can be seen that, in case I, the two torsional stiffnesses are almost the same, while in case II, the $c_{44}$ given by the axially unrestrained FEM model is about half of the restrained one, thus confirming the importance of the macro-warping effect. Finally, the numerical analysis of the cell, also corroborates the assumptions here made on the analytical identification of the torsional stiffness: indeed, the percentage error made in the analytical evaluation of the (constrained model) coefficients (remember Table 2), with respect to the numerical ones, is about $2 \%$ in both the case studies. 


\subsection{Dynamics}

The free dynamics of the grid beam is now analyzed. By letting $\mathbf{p}=\mathbf{0}$ and $\mathbf{u}=\hat{\mathbf{u}}(s) e^{\lambda t}$ in Equation (5), $(\lambda, \hat{\mathbf{u}}(s))$ being the eigenpairs of the system, a (spatial) boundary eigenvalue problem is obtained. Since the problem is conservative, the eigenvalues are purely imaginary, namely, $\lambda_{j}= \pm i \omega_{j}$, where $\omega_{j}$ is the angular frequency of the $j$-th mode. As already discussed, the boundary eigenvalue problem admits an exact solution, which, in what follows is compared with FE results, to check the effectiveness of the homogenized beam model in capturing frequencies and modal shapes of the real grid beam.

Comparison is relevant to the first six modes of the fine and coarse models and it is made for all the case studies, already discussed in statics. The results of modal analysis are presented in Table 3, where the frequencies, $\omega_{E Q}$ and $\omega_{F E}$, relevant to the equivalent beam model and to FE solutions, respectively, are reported; moreover, $\epsilon_{\%}=100\left(\omega_{F E}-\omega_{E Q}\right) / \omega_{F E}$ is the percentage error. It is seen that the equivalent beam model furnishes a very accurate approximation of the first six frequencies of the fine model, particularly in the case studies I-a $(n=40,100)$ and I-b $(n=40)$, where the maximum error is about $5 \%$. Otherwise, in case study II-a, the percentage error increases, where just for the torsional modes (up to about $20 \%$ ), this behavior is in agreement with what was already discussed in the static analysis.

Finally, a very good accordance is confirmed also by analyzing the the modal shapes of the first six modes in case studies I-a $(n=40,100)$, and I-b $(n=40)$, reported in Figures 9-11, respectively.

Table 3. Angular frequencies of the first six modes for all the case studies: $\omega_{E Q}, \omega_{F E}$ refer to Equivalent Beam model and FE solutions, respectively.

\begin{tabular}{|c|c|c|c|c|c|}
\hline Case Study & Mode & Description (F = Flexural, $\mathrm{T}=$ Torsional $)$ & $\begin{array}{l}\omega_{E Q} \\
{[\mathrm{rad}]}\end{array}$ & $\begin{array}{l}\omega_{F E} \\
{[\mathrm{rad}]}\end{array}$ & $\begin{array}{c}\epsilon \% \\
{[-]}\end{array}$ \\
\hline \multirow{4}{*}{$\mathrm{I}-\mathrm{a}, n=40$} & 1,2 & 1st $\mathrm{F}(x, y)$-plane, 1 st $\mathrm{F}(x, z)$-plane & 195.58 & 188.92 & 3.5 \\
\hline & 3 & 1st $\mathrm{T}$ & 280.76 & 271.05 & 3.6 \\
\hline & 4,5 & 2nd F $(x, y)$-plane, 2nd F $(x, z)$-plane & 641.16 & 624.07 & 2.7 \\
\hline & 6 & 2nd T & 842.27 & 816.63 & 3.1 \\
\hline \multirow{4}{*}{$\mathrm{I}-\mathrm{a}, n=100$} & 1,2 & 1st $\mathrm{F}(x, y)$-plane, 1 st $\mathrm{F}(x, z)$-plane & 42.82 & 42.16 & 1.6 \\
\hline & 3 & 1st $\mathrm{T}$ & 112.30 & 107.81 & 4.2 \\
\hline & 4,5 & 2nd F $(x, y)$-plane, 2nd $\mathrm{F}(x, z)$-plane & 192.60 & 187.28 & 2.8 \\
\hline & 6 & 2nd T & 336.91 & 324.03 & 4.0 \\
\hline \multirow{4}{*}{$\mathrm{I}-\mathrm{b}, n=40$} & 1,2 & 1st $\mathrm{F}(x, y)$-plane, 1st $\mathrm{F}(x, z)$-plane & 437.76 & 433.86 & 0.9 \\
\hline & 3 & 1st $\mathrm{T}$ & 561.51 & 557.96 & 0.6 \\
\hline & 4,5 & 2nd F $(x, y)$-plane, 2nd F $(x, z)$-plane & 988.03 & 979.15 & 0.9 \\
\hline & 6 & 2nd T & 1123.03 & 1119.60 & 0.3 \\
\hline \multirow{6}{*}{ II-a, $n=40$} & 1 & 1st $\mathrm{F}(x, y)$-plane & 116.03 & 113.80 & 2.0 \\
\hline & 2 & 1st $\mathrm{F}(x, z)$-plane & 211.03 & 203.81 & 3.5 \\
\hline & 3 & 1 st $\mathrm{T}$ & 287.47 & 238.38 & 20.1 \\
\hline & 4 & 2nd F $(x, y)$-plane & 503.20 & 496.07 & 1.4 \\
\hline & 5 & 2nd $\mathrm{F}(x, z)$-plane & 693.98 & 673.15 & 3.1 \\
\hline & 6 & 2nd T & 862.42 & 743.75 & 16.0 \\
\hline
\end{tabular}




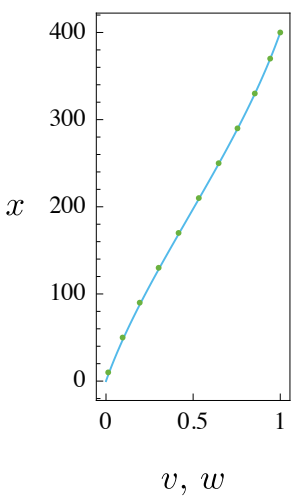

(a)

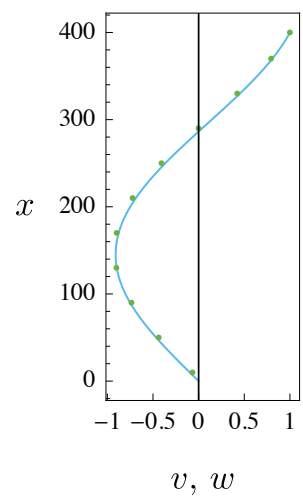

(b)

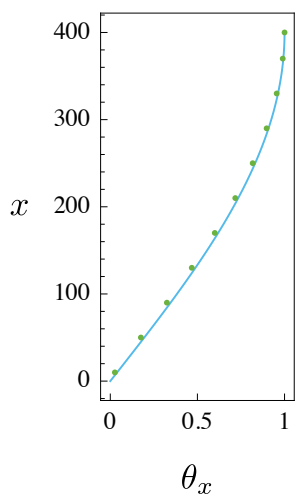

(c)

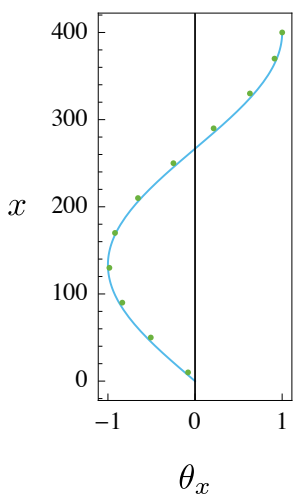

(d)

Figure 9. Modal shapes of the equivalent model vs. discrete FE model, for case study I-a and $n=40$ : $(\mathbf{a}, \mathbf{b})$ First and second flexural mode; (c,d) first and second torsional mode. Green dots: discrete FE solution. Continuous blue line: homogenized beam model.

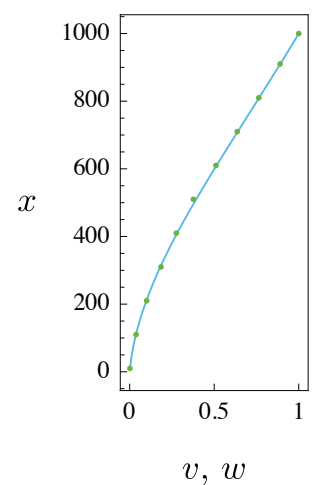

(a)

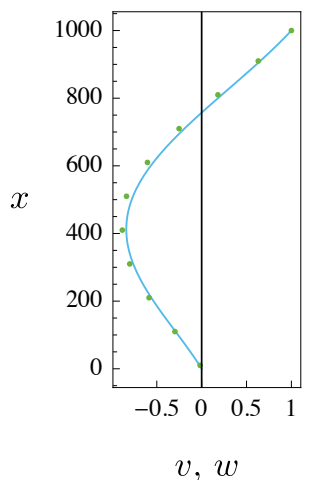

(b)

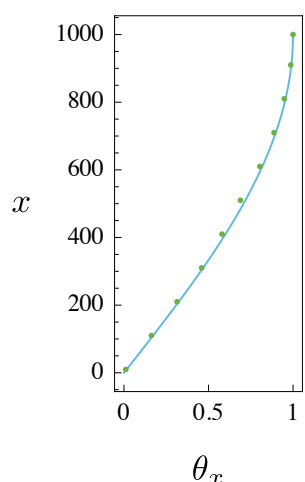

(c)

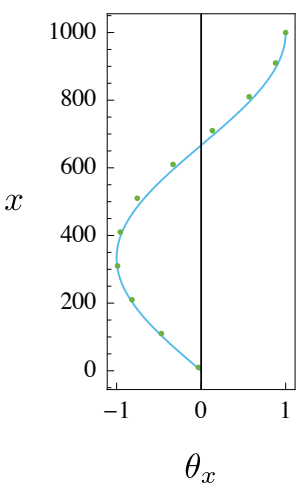

(d)

Figure 10. Modal shapes of the equivalent model vs. discrete FE model, for case study I-a and $n=100$ : $(\mathbf{a}, \mathbf{b})$ First and second flexural mode; (c,d) first and second torsional mode. Green dots: discrete FE solution. Continuous blue line: homogenized beam model.

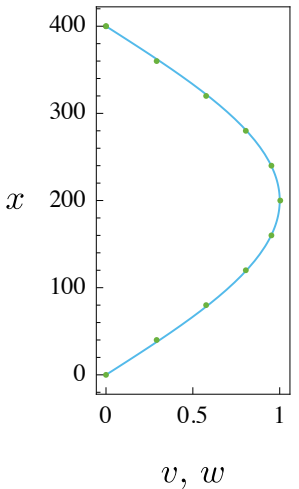

(a)

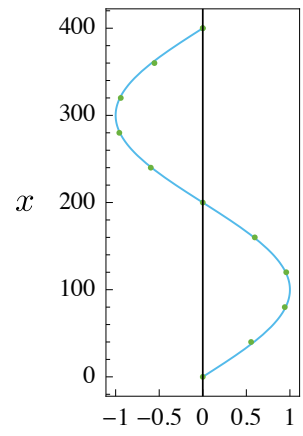

(b)

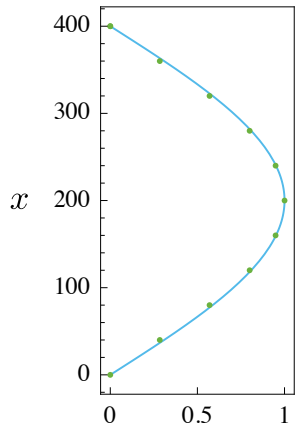

$\theta_{x}$

(c)

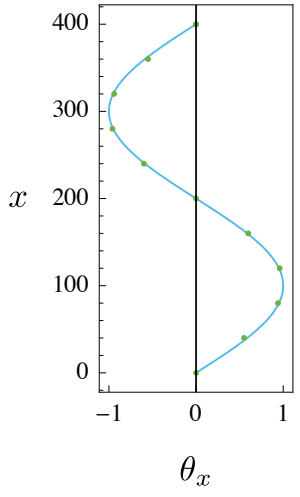

(d)

Figure 11. Modal shapes of the equivalent model vs. discrete FE model, for case study I-b and $n=40$ : $(\mathbf{a}, \mathbf{b})$ First and second flexural mode; (c,d) first and second torsional mode. Green dots: discrete FE solution. Continuous blue line: homogenized beam model.

\section{Conclusions and Perspectives}

Static and dynamic analyses of grid beams have been addressed in the present paper. To this end, a Timoshenko beam model was formulated in the framework of direct 1D modeling. Its constitutive 
law was determined via a homogenization procedure, based on the energy equivalence between the cell of the periodic grid beam and a segment of the equivalent beam, under the same point-wise displacement fields. The presented model also incorporates corrective factors, analytically determined, which accounts, on average, for the micro-warping effect. Moreover, inertial properties of the coarse model have been determined, under the assumption of lumped masses at the joints.

The effectiveness and limits of applicability of the Timoshenko beam model have been discussed by numerical analyses, carried out on sample grid beams, taken as case studies. Numerical results, concerning the exact solution of the coarse model in terms of static response and modal properties, have been compared with Finite-Element analyses.

The following conclusions have been drawn.

1. An excellent accuracy in terms of translational displacements is given by the Timoshenko model, both in statics and in free dynamics (modal shapes), independently from the number of cells.

2. An excellent agreement between coarse and fine models is also detected, both in statics and in free dynamics (modal shapes), for torsional displacements, where when the aspect ratio of the grid beam cross-section is close to 1 , this circumstance reduces the effect of macro-warping. In contrast, when the cross-section is thin, significant errors due to macro-warping appear.

3. Very good agreement is also detected in terms of natural frequencies. In this case, the macro-warping effect also entails worsening of the accuracy in the torsional modes.

The main perspective of this work relies, therefore, on the development of direct 1D models of beams, and is able to take into account the effects of macro-warping and to investigate the apparent similarity existing between solid and grid beams, when the aspect ratio is far from 1 .

Author Contributions: Conceptualization, A.L.; methodology, F.D., M.F. and A.L.; software, F.D. and M.F.; validation, F.D., M.F. and A.L.; data curation, F.D. and M.F.; writing-original draft preparation, F.D., M.F. and A.L.; writing-review and editing, F.D., M.F. and A.L. All authors have read and agreed to the published version of the manuscript.

Funding: This research received no external funding.

Conflicts of Interest: The authors declare no conflict of interest.

\section{References}

1. Maconachie, T.; Leary, M.; Lozanovski, B.; Zhang, X.; Qian, M.; Faruque, O.; Brandt, M. SLM lattice structures: Properties, performance, applications and challenges. Mater. Des. 2019, 183, 108137. [CrossRef]

2. Mieszala, M.; Hasegawa, M.; Guillonneau, G.; Bauer, J.; Raghavan, R.; Frantz, C.; Kraft, O.; Mischler, S.; Michler, J.; Philippe, L. Micromechanics of amorphous metal/polymer hybrid structures with 3D cellular architectures: Size effects, buckling behavior, and energy absorption capability. Small 2017, 13, 1602514. [CrossRef]

3. Casalotti, A.; D'Annibale, F.; Rosi, G. Multi-scale design of an architected composite structure with optimized graded properties. Compos. Struct. 2020, 252, 112608. [CrossRef]

4. Askar, A.; Cakmak, A.S. A structural model of a micropolar continuum. Int. J. Eng. Sci. 1968, 6, 583-589. [CrossRef]

5. Dos Reis, F.; Ganghoffer, J.F. Construction of micropolar continua from the asymptotic homogenization of beam lattices. Comput. Struct. 2012, 112, 354-363. [CrossRef]

6. Noor, A.K. Continuum modeling for repetitive lattice structures. Appl. Mech. Rev. 1988, 41, $285-296$. [CrossRef]

7. Tollenaere, H.; Caillerie, D. Continuous modeling of lattice structures by homogenization. Adv. Eng. Softw. 1998, 29, 699-705. [CrossRef]

8. Di Nino, S.; Luongo, A. A simple homogenized orthotropic model for in-plane analysis of regular masonry walls. Int. J. Solids Struct. 2019, 167, 156-169. [CrossRef]

9. Hache, F.; Challamel, N.; Elishakoff, I.; Wang, C. Comparison of nonlocal continualization schemes for lattice beams and plates. Arch. Appl. Mech. 2017, 87, 1105-1138. [CrossRef] 
10. Boutin, C.; Hans, S.; Chesnais, C. Generalized Beams and Continua. Dynamics of Reticulated Structures. In Mechanics of Generalized Continua: One Hundred Years After the Cosserats; Maugin, G.A., Metrikine, A.V., Eds.; Springer: New York, NY, USA, 2010; pp. 131-141.

11. Boutin, C.; dell'Isola, F.; Giorgio, I.; Placidi, L. Linear pantographic sheets: Asymptotic micro-macro models identification. Math. Mech. Complex Syst. 2017, 5, 127-162. [CrossRef]

12. dell'Isola, F.; Eremeyev, V.A.; Porubov, A. Advances in Mechanics of Microstructured Media and Structures; Springer: Cham, Switzerland, 2018; Volume 87.

13. dell'Isola, F.; Seppecher, P.; Spagnuolo, M.; Barchiesi, E.; Hild, F.; Lekszycki, T.; Giorgio, I.; Placidi, L.; Andreaus, U.; Cuomo, M.; et al. Advances in pantographic structures: Design, manufacturing, models, experiments and image analyses. Contin. Mech. Thermodyn. 2019, 31, 1231-1282. [CrossRef]

14. dell'Isola, F.; Seppecher, P.; Alibert, J.J.; Lekszycki, T.; Grygoruk, R.; Pawlikowski, M.; Steigmann, D.; Giorgio, I.; Andreaus, U.; Turco, E.; et al. Pantographic metamaterials: An example of mathematically driven design and of its technological challenges. Contin. Mech. Thermodyn. 2019, 31, 851-884. [CrossRef]

15. Giorgio, I.; Rizzi, N.L.; Turco, E. Continuum modelling of pantographic sheets for out-of-plane bifurcation and vibrational analysis. Proc. R. Soc. A Math. Phys. Eng. Sci. 2017, 473, 20170636. [CrossRef]

16. De Angelo, M.; Barchiesi, E.; Giorgio, I.; Abali, B.E. Numerical identification of constitutive parameters in reduced-order bi-dimensional models for pantographic structures: Application to out-of-plane buckling. Arch. Appl. Mech. 2019, 89, 1333-1358. [CrossRef]

17. Challamel, N.; Hache, F.; Elishakoff, I.; Wang, C. Buckling and vibrations of microstructured rectangular plates considering phenomenological and lattice-based nonlocal continuum models. Compos. Struct. 2016, 149, 145-156. [CrossRef]

18. Hache, F.; Challamel, N.; Elishakoff, I. Lattice and continualized models for the buckling study of nonlocal rectangular thick plates including shear effects. Int. J. Mech. Sci. 2018, 145, 221-230. [CrossRef]

19. Chajes, M.J.; Romstad, K.M.; McCallen, D.B. Analysis of multiple-bay frames using continuum model. J. Struct. Eng. 1993, 119, 522-546. [CrossRef]

20. Zalka, K.A. Global Structural Analysis of Buildings; CRC Press: Boca Raton, FL, USA, 2002.

21. Potzta, G.; Kollár, L. Analysis of building structures by replacement sandwich beams. Int. J. Solids Struct. 2003, 40, 535-553. [CrossRef]

22. Boutin, C.; Hans, S. Homogenisation of periodic discrete medium: Application to dynamics of framed structures. Comput. Geotech. 2003, 30, 303-320. [CrossRef]

23. Hans, S.; Boutin, C. Dynamics of discrete framed structures: A unified homogenized description. J. Mech. Mater. Struct. 2008, 3, 1709-1739. [CrossRef]

24. Chesnais, C.; Boutin, C.; Hans, S. Structural Dynamics and Generalized Continua. In Mechanics of Generalized Continua; Altenbach, H., Maugin, G., Erofeev, V., Eds.; Springer: Berlin/Heidelberg, Germany, 2011; pp. 57-76.

25. Luongo, A. Statics, Dynamics, Buckling and Aeroelastic Stability of Planar Cellular Beams. In Modern Trends in Structural and Solid Mechanics; Challamel, N., Kaplunov, J., Takewaki, I., Eds.; ISTE-Wiley: Hoboken, NJ, USA, 2020.

26. Piccardo, G.; Tubino, F.; Luongo, A. A shear-shear torsional beam model for nonlinear aeroelastic analysis of tower buildings. Z. Für Angew. Math. Und Phys. 2015, 66, 1895-1913. [CrossRef]

27. Piccardo, G.; Tubino, F.; Luongo, A. Equivalent nonlinear beam model for the 3-D analysis of shear-type buildings: Application to aeroelastic instability. Int. J. Non-Linear Mech. 2016, 80, 52-65. [CrossRef]

28. Piccardo, G.; Tubino, F.; Luongo, A. Equivalent Timoshenko linear beam model for the static and dynamic analysis of tower buildings. Appl. Math. Model. 2019, 71,77-95. [CrossRef]

29. Ferretti, M. Flexural torsional buckling of uniformly compressed beam-like structures. Contin. Mech. Thermodyn. 2018, 30, 977-993. [CrossRef]

30. D'Annibale, F.; Ferretti, M.; Luongo, A. Shear-shear-torsional homogenous beam models for nonlinear periodic beam-like structures. Eng. Struct. 2019, 184, 115-133. [CrossRef]

31. Di Nino, S.; Luongo, A. Nonlinear aeroelastic behavior of a base-isolated beam under steady wind flow. Int. J. Non-Linear Mech. 2020, 119, 103340. [CrossRef]

32. Ferretti, M.; D'Annibale, F.; Luongo, A. Buckling of tower-buildings on elastic foundation under compressive tip-forces and self-weight. Contin. Mech. Thermodyn. 2020. [CrossRef] 
33. Luongo, A.; Zulli, D. Free and forced linear dynamics of a homogeneous model for beam-like structures. Meccanica 2020, 55, 907-925. [CrossRef]

34. Luongo, A.; D'Annibale, F.; Ferretti, M. Shear and flexural factors for homogenized beam models of planar frames. Eng. Struct. 2020, submitted.

35. Ferretti, M.; D'Annibale, F.; Luongo, A. Modeling beam-like planar structures by a one-dimensional continuum: an analytical-numerical method. J. Appl. Comput. Mech. 2020, in press.

36. Eugster, S.; Steigmann, D. Continuum theory for mechanical metamaterials with a cubic lattice substructure. Math. Mech. Complex Syst. 2019, 7, 75-98. [CrossRef]

37. Antman, S.S. The theory of rods. In Linear Theories of Elasticity and Thermoelasticity; Springer: Berlin/Heidelberg, Germany, 1973; pp. 641-703.

38. Antman, S.S. Nonlinear Problems of Elasticity; Springer: New York, NY, USA, 2005.

39. Capriz, G. A Contribution to the Theory of Rods. Riv. Mat. Univ. Parma 1981, 7, 489-506.

40. Luongo, A.; Zulli, D. Mathematical Models of Beams and Cables; John Wiley \& Sons: Hoboken, NJ, USA, 2013.

41. Altenbach, H.; Bîrsan, M.; Eremeyev, V.A. Cosserat-type rods. In Generalized Continua from the Theory to Engineering Applications; Springer: Wien, Austria, 2013; pp. 179-248.

42. Steigmann, D.J.; Faulkner, M.G. Variational theory for spatial rods. J. Elast. 1993, 33, 1-26. [CrossRef]

43. Elishakoff, I. Handbook on Timoshenko-Ehrenfest Beam and Uflyand-Mindlin Plate Theories; World Scientific: Singapore, 2020.

44. Cazzani, A.; Stochino, F.; Turco, E. On the whole spectrum of Timoshenko beams. Part I: A theoretical revisitation. Z. Für Angew. Math. Und Phys. 2016, 67, 24. [CrossRef]

45. Cazzani, A.; Stochino, F; Turco, E. On the whole spectrum of Timoshenko beams. Part II: Further applications. Z. Für Angew. Math. Und Phys. 2016, 67, 25. [CrossRef]

46. Silvestre, N.; Camotim, D. First-order generalised beam theory for arbitrary orthotropic materials. Thin-Walled Struct. 2002, 40, 755-789. [CrossRef]

47. Ranzi, G.; Luongo, A. A new approach for thin-walled member analysis in the framework of GBT. Thin-Walled Struct. 2011, 49, 1404-1414. [CrossRef]

48. Taig, G.; Ranzi, G.; D'Annibale, F. An unconstrained dynamic approach for the generalised beam theory. Contin. Mech. Thermodyn. 2015, 27, 879-904. [CrossRef]

(C) 2020 by the authors. Licensee MDPI, Basel, Switzerland. This article is an open access article distributed under the terms and conditions of the Creative Commons Attribution (CC BY) license (http:/ / creativecommons.org/licenses/by/4.0/). 